\title{
The centers of gravity of the associahedron and of the permutahedron are the same
}

\author{
Christophe Hohlweg * \\ LaCIM et Département de Mathématiques \\ Université du Québec à Montréal \\ CP 8888 Succ. Centre-Ville \\ Montréal, Québec, H3C 3P8 CANADA \\ hohlweg. christophe@uqam.ca
}

\author{
Jonathan Lortie \\ LaCIM et Département de Mathématiques \\ Université du Québec à Montréal \\ CP 8888 Succ. Centre-Ville \\ Montréal, Québec, H3C 3P8 CANADA \\ lortie.jonathan@courrier.uqam.ca
}

\author{
Annie Raymond \\ Berlin Mathematical School \\ Strasse des 17. Juni 136 \\ Berlin, 10623, Germany \\ raymond@math.tu-berlin.de
}

Submitted: Mar 8, 2010; Accepted: May 4, 2010; Published: May 14, 2010

Mathematics Subject Classification: 05E18;05E99

\begin{abstract}
In this article, we show that Loday's realization of the associahedron has the the same center of gravity as the permutahedron. This proves an observation made by F. Chapoton.

We also prove that this result holds for the associahedron and the cyclohedron as realized by the first author and C. Lange.
\end{abstract}

\section{Introduction.}

This article is the continuation of previous work $[7,8,1]$ devoted to the study of generalized associahedra via geometric and combinatorial tools arising from finite Coxeter groups.

In 1963, J. Stasheff discovered a cell complex [21, 22] of great importance in algebraic topology, geometric topology and combinatorics $([2,6,10])$. This cell complex can be realized as a simple $n-1$-dimensional convex polytope in $\mathbb{R}^{n}$ : the associahedron. Many realizations of the associahedron were given over the last thirty years ([13]). In

*This work is supported by FQRNT and NSERC. It is the result of a summer undergraduate research internship supported by LaCIM

THE EleCtronic Journal of COMBinatorics 17 (2010), \#R72 
2004, J. L. Loday ([9]) computed the classical realization of the associahedron given by S. Shnider and S. Sternberg in [19]. Loday gave a beautiful combinatorial algorithm to compute the integer coordinates of the vertices of the associahedron, and showed that his realization can be obtained naturally from the classical permutahedron of dimension $n-1$, i.e. the convex hull of all possible permutations of the point $(1, \ldots, n)$ in $\mathbb{R}^{n}$. The permutahedron encodes the combinatorics and the geometry behind the symmetric group $S_{n}$. The main motivation behind Loday's realization is to study the geometric and combinatorial properties of the associahedron via those of the well-known permutahedron.

Among the many areas of mathematics where the associahedron appears, cluster algebra and Cambrian fans are the ones motivating our work. In 2000, S. Fomin and A. Zelevinsky introduced a new family of fans indexed by Weyl groups called cluster fans, whose structure encodes the one of finitely generated cluster algebras [5]. These fans are polytopal and the corresponding polytopes, called generalized associahedra, have been first realized by F. Chapoton, S. Fomin and A. Zelevinsky in [4]. In this realization, not only is the face structure of generalized associahedra relevant, but so is their geometry. It turned out that generalized associahedra associated to symmetric groups are combinatorially isomorphic to Stasheff's associahedra, but different from Loday's realization.

In 2006, N. Reading came up with an elegant framework that suggested how to study generalized associahedra via the theory of Coxeter groups, a superclass of Weyl groups. The key objects introduced by N. Reading are called Cambrian fans and lattices $[14,15$, 16, 17]. For each orientation of the Coxeter graph of a given finite Coxeter group, there is a Cambrian fan which provides a combinatorial and geometric interpretation of cluster fans as well as an explanation of their links with quiver theory as discussed by R. Marsh, M. Reineke and A. Zelevinsky in [12].

The discovery of polytopal realizations of Cambrian fans built from $W$-permutahedra [8] strengthened the relationship between Coxeter groups theory and Cambrian theory. It is now worth mentioning that the permutahedron can be realized for any finite Coxeter group $W$ simply by taking the convex hull of the orbit of a generic point under the reflective action of $W$. These realizations enjoy two important properties. First, they generalize Loday's realization to any finite Coxeter group as they are obtained by simply removing some of the defining halfspaces of the $W$-permutahedron (Figure 1). Second, they provide combinatorial and geometric interpretations of cluster fans and their links to quiver theory since their normal fans are of Cambrian nature. We name these realizations $c$-generalized associahedra, where $c$ refers to the orientation of the Coxeter graph of $W$.

We investigate the following question : what are the geometric properties that are preserved when we construct a $c$-generalized associahedron from a given $W$-permutahedron? We believe that the answer to this question would help us to highlight and refine the links between finite Coxeter groups theory and Cambrian theory. Except for the isometry classes of these realizations [1], little is known about them. What are their volumes? The number of integer points they contain? Their isometry groups?

It has been observed in numerous and large example (see [7, 8]) that the center of gravity remains unchanged whatever the orientation is chosen to be. J. L. Loday already reported in [9, Section 2.11] an observation made by F. Chapoton that the centers of 

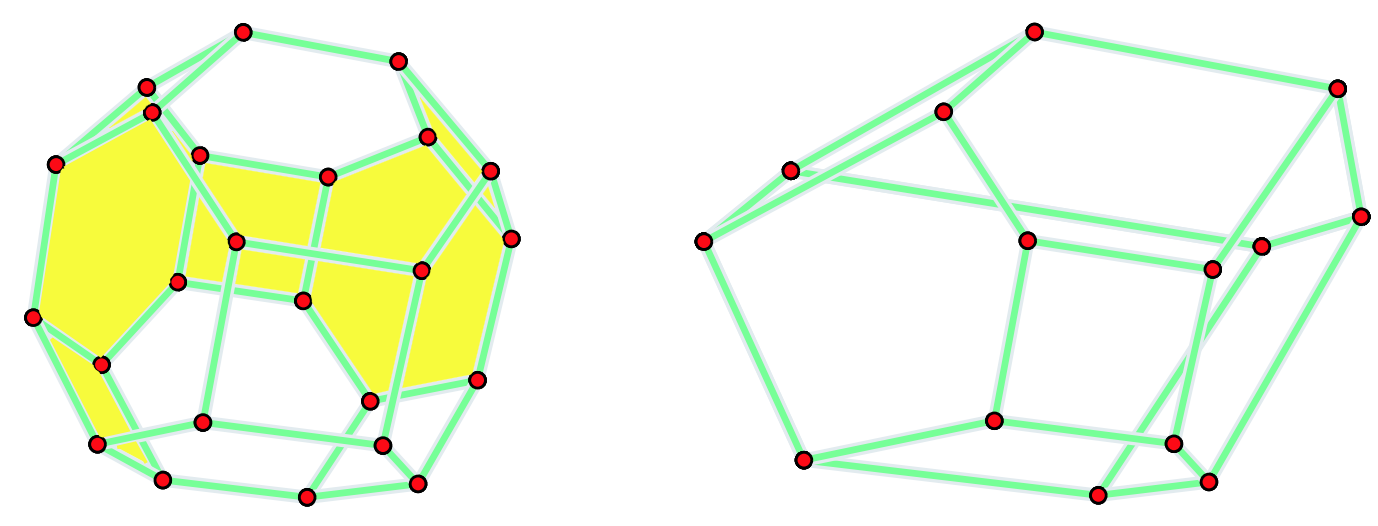

Figure 1: We obtain the associahedron (right) from the permutahedron (left) for the Coxeter group $S_{4}$ and the left-to-right orientation by removing all shaded halfspaces.

gravity of the vertices of the associahedron and of the permutahedron are the same. No proof is given, and after asking both F. Chapoton and J. L. Loday, it seems that this property of Loday's realization has never been proven until now. For symmetric groups (type A) and hyperoctahedral groups (type B), c-generalized associahedra were first realized by C. Hohlweg and C. Lange [7], a realization that recovers Loday's for a particular choice of orientation of the Coxeter graph of type A.

In this article we prove that for type $\mathrm{A}$ and $\mathrm{B}$, the center of gravity remains unchanged when we remove halfspaces from the permutahedron to build a $c$-generalized associahedron.

The center of gravity (also known as isobarycenter or centroid) is a classical invariant of configuration of points whose significance in mechanical physics and classical euclidean geometry is not disputed. It also appears as a powerful tool in computational geometry and computer science (see $[18,11]$ ).

Our result highlights an interesting partition of the set of vertices of $c$-generalized associahedra. A classical way to prove that a point is the center of gravity is to find enough isometries of the polytope whose axes intersect in this point. Unfortunately, already in the case $A_{2}$, there is only one nontrivial isometry of $c$-generalized associahedra, so we may only conclude that the center of gravity takes place on this axe. In order to overcome this problem, we find a partition of the set of vertices, which are parameterized in this case by the triangulations of a regular polygon, and by their isometry classes under the action of the corresponding dihedral group. Then, we show that the center of gravity for each of these classes is the same as the center of gravity of the permutahedron. For the reader familiar with Cambrian fans and $c$-clusters, we want to note that preliminary computations on general cases where the vertices are parameterized by $c$-clusters, allow us to observe the same phenomenon and identify orbits of a particular nonlinear action of a group on subsets of almost positive roots. The study of this group and its action will be the subject of future publications. 
The article is organized as follows. In $\S 2$, we first recall the realization of the permutahedron and how to compute its center of gravity. Then we compute the center of gravity of Loday's realization of the associahedron. In order to do this, we partition its vertices into isometry classes of triangulations, which parameterize the vertices, and we show that the center of gravity for each of those classes is the center of gravity of the permutahedron.

In $\S 3$, we show that the computation of the center of gravity of any of the realizations given by the first author and $\mathrm{C}$. Lange is reduced to the computation of the center of gravity of the Loday's classical realization of the associahedron. We do the same for the cyclohedron in $\S 4$.

We are grateful to Carsten Lange for allowing us to use some of the pictures he made in $[7]$.

\section{Center of gravity of the classical permutahedron and associahedron}

\subsection{The permutahedron}

Let $S_{n}$ be the symmetric group acting on the set $[n]=\{1,2, \ldots, n\}$. The permutahedron $\operatorname{Perm}\left(S_{n}\right)$ is the classical $n$-1-dimensional simple convex polytope defined as the convex hull of the points

$$
M(\sigma)=(\sigma(1), \sigma(2), \ldots, \sigma(n)) \in \mathbb{R}^{n}, \quad \forall \sigma \in S_{n}
$$

The center of gravity (or isobarycenter) is the unique point $G$ of $\mathbb{R}^{n}$ such that

$$
\sum_{\sigma \in S_{n}} \overrightarrow{G M(\sigma)}=\overrightarrow{0}
$$

Since the permutation $w_{0}: i \mapsto n+1-i$ preserves $\operatorname{Perm}\left(S_{n}\right)$, we see, by sending $M(\sigma)$ to

$$
M\left(w_{0} \sigma\right)=(n+1-\sigma(1), n+1-\sigma(2), \ldots, n+1-\sigma(n)),
$$

that the center of gravity is $G=\left(\frac{n+1}{2}, \frac{n+1}{2}, \ldots, \frac{n+1}{2}\right)$.

\subsection{Loday's realization}

We present here the realization of the associahedron given by J. L. Loday [9]. However, instead of using planar binary trees, we use triangulations of a regular polygon to parameterize the vertices of the associahedron (see [7, Remark 1.2]). 


\subsubsection{Triangulations of a regular polygon}

Let $P$ be a regular $(n+2)$-gon in the Euclidean plane with vertices $A_{0}, A_{1}, \ldots, A_{n+1}$ in counterclockwise direction. A triangulation of $P$ is a set of $n$ noncrossing diagonals of $P$.

Let us be more explicit. A triangle of $P$ is a triangle whose vertices are vertices of $P$. Therefore a side of a triangle of $P$ is either an edge or a diagonal of $P$. A triangulation of $P$ is then a collection of $n$ distinct triangles of $P$ with noncrossing sides. Any of the triangles in $T$ can be described as $A_{i} A_{j} A_{k}$ with $0 \leqslant i<j<k \leqslant n+1$. Each $1 \leqslant j \leqslant n$ corresponds to a unique triangle $\Delta_{j}(T)$ in $T$ because the sides of triangles in $T$ are noncrossing.

Therefore we write $T=\left\{\Delta_{1}(T), \ldots, \Delta_{n}(T)\right\}$ for a triangulation $T$, where $\Delta_{j}(T)$ is the unique triangle in $T$ with vertex $A_{j}$ and the two other vertices $A_{i}$ and $A_{k}$ satisfying the inequation $0 \leqslant i<j<k \leqslant n+1$.

Denote by $\mathcal{T}_{n+2}$ the set of triangulations of $P$.

\subsubsection{Loday's realization of the associahedron}

Let $T$ be a triangulation of $P$. The weight $\delta_{j}(T)$ of the triangle $\Delta_{j}(T)=A_{i} A_{j} A_{k}$, where $i<j<k$, is the positive number

$$
\delta_{j}(T)=(j-i)(k-j)
$$

The weight $\delta_{j}(T)$ of $\Delta_{j}(T)$ represents the product of the number of boundary edges of $P$ between $A_{i}$ and $A_{j}$ passing through vertices indexed by smaller numbers than $j$ with the number of boundary edges of $P$ between $A_{j}$ and $A_{k}$ passing through vertices indexed by larger numbers than $j$.

The classical associahedron $\operatorname{Asso}\left(S_{n}\right)$ is obtained as the convex hull of the points

$$
M(T)=\left(\delta_{1}(T), \delta_{2}(T), \ldots, \delta_{n}(T)\right) \in \mathbb{R}^{n}, \quad \forall T \in \mathcal{T}_{n+2} .
$$

We are now able to state our first result.

Theorem 2.1. The center of gravity of $\operatorname{Asso}\left(S_{n}\right)$ is $G=\left(\frac{n+1}{2}, \frac{n+1}{2}, \ldots, \frac{n+1}{2}\right)$.

In order to prove this theorem, we need to study closely a certain partition of the vertices of $P$.

\subsection{Isometry classes of triangulations}

As $P$ is a regular $(n+2)$-gon, its isometry group is the dihedral group $\mathcal{D}_{n+2}$ of order $2(n+2)$. So $\mathcal{D}_{n+2}$ acts on the set $\mathcal{T}_{n+2}$ of all triangulations of $P$ : for $f \in \mathcal{D}_{n+2}$ and $T \in \mathcal{T}_{n+2}$, we have $f \cdot T \in \mathcal{T}_{n+2}$. We denote by $\mathcal{O}(T)$ the orbit of $T \in \mathcal{T}_{n+2}$ under the action of $\mathcal{D}_{n+2}$.

We know that $G$ is the center of gravity of $\operatorname{Asso}\left(S_{n}\right)$ if and only if

$$
\sum_{T \in \mathcal{T}_{n+2}} \overrightarrow{G M(T)}=\overrightarrow{0}
$$


As the orbits of the action of $\mathcal{D}_{n+2}$ on $\mathcal{T}_{n+2}$ form a partition of the set $\mathcal{T}_{n+2}$, it is sufficient to compute

$$
\sum_{T \in \mathcal{O}} \overrightarrow{G M(T)}
$$

for any orbit $\mathcal{O}$. The following key observation implies directly Theorem 2.1.

Theorem 2.2. Let $\mathcal{O}$ be an orbit of the action of $\mathcal{D}_{n+2}$ on $\mathcal{T}_{n+2}$, then $G$ is the center of gravity of $\{M(T) \mid T \in \mathcal{O}\}$. In particular, $\sum_{T \in \mathcal{O}} \overrightarrow{G M(T)}=\overrightarrow{0}$.

Before proving this theorem, we need to prove the following result.

Proposition 2.3. Let $T \in \mathcal{T}_{n+2}$ and $j \in[n]$, then $\sum_{f \in \mathcal{D}_{n+2}} \delta_{j}(f \cdot T)=(n+1)(n+2)$.

Proof. We prove this proposition by induction on $j \in[n]$. For any triangulation $T^{\prime}$, we denote by $a_{j}\left(T^{\prime}\right)<j<b_{j}\left(T^{\prime}\right)$ the indices of the vertices of $\Delta_{j}\left(T^{\prime}\right)$. Let $H$ be the group of rotations in $\mathcal{D}_{n+2}$. It is well-known that for any reflection $s \in \mathcal{D}_{n+2}$, the classes $H$ and $s H$ form a partition of $\mathcal{D}_{n+2}$ and that $|H|=n+2$. We consider also the unique reflection $s_{k} \in \mathcal{D}_{n+2}$ which maps $A_{x}$ to $A_{n+3+k-x}$, where the values of the indices are taken in modulo $n+2$. In particular, $s_{k}\left(A_{0}\right)=A_{n+3+k}=A_{k+1}, s_{k}\left(A_{1}\right)=A_{k}, s_{k}\left(A_{k+1}\right)=A_{n+2}=A_{0}$, and so on.

Basic step $j=1$ : We know that $a_{1}\left(T^{\prime}\right)=0$ for any triangulation $T^{\prime}$, hence the weight of $\Delta_{1}\left(T^{\prime}\right)$ is $\delta_{1}\left(T^{\prime}\right)=(1-0)\left(b_{1}\left(T^{\prime}\right)-1\right)=b_{1}\left(T^{\prime}\right)-1$.

The reflection $s_{0} \in \mathcal{D}_{n+2}$ maps $A_{x}$ to $A_{n+3-x}$ (where $A_{n+2}=A_{0}$ and $A_{n+3}=A_{1}$ ). In other words, $s_{0}\left(A_{0}\right)=A_{1}$ and $s_{0}\left(\Delta_{1}\left(T^{\prime}\right)\right)$ is a triangle in $s_{0} \cdot T^{\prime}$. Since

$$
s_{0}\left(\Delta_{1}\left(T^{\prime}\right)\right)=s_{0}\left(A_{0} A_{1} A_{b_{1}\left(T^{\prime}\right)}\right)=A_{0} A_{1} A_{n+3-b_{1}\left(T^{\prime}\right)}
$$

and $0<1<n+3-b_{1}\left(T^{\prime}\right), s_{0}\left(\Delta_{1}\left(T^{\prime}\right)\right)$ has to be $\Delta_{1}\left(s_{0} \cdot T^{\prime}\right)$. In consequence, we obtain that

$$
\delta_{1}\left(T^{\prime}\right)+\delta_{1}\left(s_{0} \cdot T^{\prime}\right)=\left(b_{1}\left(T^{\prime}\right)-1\right)+\left(n+3-b_{1}\left(T^{\prime}\right)-1\right)=n+1,
$$

for any triangulation $T^{\prime}$. Therefore

$$
\sum_{f \in \mathcal{D}_{n+2}} \delta_{1}(f \cdot T)=\sum_{g \in H}\left(\left(\delta_{1}(g \cdot T)+\delta_{1}\left(s_{0} \cdot(g \cdot T)\right)\right)=|H|(n+1)=(n+1)(n+2),\right.
$$

proving the initial case of the induction.

Inductive step: Assume that, for a given $1 \leqslant j<n$, we have

$$
\sum_{f \in \mathcal{D}_{n+2}} \delta_{j}(f \cdot T)=(n+1)(n+2) .
$$

We will show that

$$
\sum_{f \in \mathcal{D}_{n+2}} \delta_{j+1}(f \cdot T)=\sum_{f \in \mathcal{D}_{n+2}} \delta_{j}(f \cdot T)
$$


Let $r \in H \subseteq \mathcal{D}_{n+2}$ be the unique rotation mapping $A_{j+1}$ to $A_{j}$. In particular, $r\left(A_{0}\right)=$ $A_{n+1}$. Let $T^{\prime}$ be a triangulation of $P$. We have two cases:

Case 1. If $a_{j+1}\left(T^{\prime}\right)>0$ then $a_{j+1}\left(T^{\prime}\right)-1<j<b_{j+1}\left(T^{\prime}\right)-1$ are the indices of the vertices of the triangle $r\left(\Delta_{j+1}\left(T^{\prime}\right)\right)$ in $r \cdot T^{\prime}$. Therefore, by unicity, $r\left(\Delta_{j+1}\left(T^{\prime}\right)\right)$ must be $\Delta_{j}\left(r \cdot T^{\prime}\right)$. Thus

$$
\begin{aligned}
\delta_{j+1}\left(T^{\prime}\right) & =\left(b_{j+1}\left(T^{\prime}\right)-(j+1)\right)\left(j+1-a_{j+1}\left(T^{\prime}\right)\right) \\
& =\left(\left(b_{j+1}\left(T^{\prime}\right)-1\right)-j\right)\left(j-\left(a_{i+1}\left(T^{\prime}\right)-1\right)\right) \\
& =\delta_{j}\left(r \cdot T^{\prime}\right) .
\end{aligned}
$$

In other words:

$$
\begin{aligned}
\sum_{\substack{f \in \mathcal{D}_{n+2}, \neq \\
a_{j+1}(f \cdot T) \neq 0}} \delta_{j+1}(f \cdot T) & =\sum_{\substack{f \in \mathcal{D}_{n+2}, a_{j+1}(f \cdot T) \neq 0}} \delta_{j}(r \cdot(f \cdot T)) \\
& =\sum_{\substack{g \in \mathcal{D}_{n+2}, b_{j}(g \cdot T) \neq n+1}} \delta_{j}(g \cdot T) .
\end{aligned}
$$

Case 2. If $a_{j+1}\left(T^{\prime}\right)=0$, then $j<b_{j+1}\left(T^{\prime}\right)-1<n+1$ are the indices of the vertices of $r\left(\Delta_{j+1}\left(T^{\prime}\right)\right)$, which is therefore not $\Delta_{j}\left(r \cdot T^{\prime}\right)$ : it is $\Delta_{b_{j+1}\left(T^{\prime}\right)-1}\left(r \cdot T^{\prime}\right)$. To handle this, we need to use the reflections $s_{j}$ and $s_{j-2}$.

On one hand, observe that $j+1<n+3+j-b_{j+1}\left(T^{\prime}\right)$ because $b_{j+1}\left(T^{\prime}\right)<n+1$. Therefore

$$
s_{j}\left(\Delta_{j+1}\left(T^{\prime}\right)\right)=A_{j+1} A_{0} A_{n+3+j-b_{j+1}\left(T^{\prime}\right)}=\Delta_{j+1}\left(s_{j} \cdot T^{\prime}\right) .
$$

Hence

$$
\begin{aligned}
\delta_{j+1}\left(T^{\prime}\right)+\delta_{j+1}\left(s_{j} \cdot T^{\prime}\right)= & (j+1)\left(b_{j+1}\left(T^{\prime}\right)-(j+1)\right) \\
& +(j+1)\left(n+3+j-b_{j+1}\left(T^{\prime}\right)-(j+1)\right) \\
= & (j+1)(n+1-j) .
\end{aligned}
$$

On the other hand, consider the triangle $\Delta_{j}\left(r \cdot T^{\prime}\right)$ in $r \cdot T^{\prime}$. Since

$$
r\left(\Delta_{j+1}\left(T^{\prime}\right)\right)=A_{j} A_{b_{j+1}\left(T^{\prime}\right)-1} A_{n+1}=\Delta_{b_{j+1}\left(T^{\prime}\right)-1}\left(r \cdot T^{\prime}\right)
$$

is in $r \cdot T^{\prime},[j, n+1]$ is a diagonal in $r \cdot T^{\prime}$. Hence $b_{j}\left(r \cdot T^{\prime}\right)=n+1$. Thus $\Delta_{j}\left(r \cdot T^{\prime}\right)=$ $A_{a_{j}\left(r \cdot T^{\prime}\right)} A_{j} A_{n+1}$ and $\delta_{j}\left(r \cdot T^{\prime}\right)=\left(j-a_{j}\left(r \cdot T^{\prime}\right)\right)(n+1-j)$. We have $s_{j-2}\left(A_{j}\right)=A_{n+1}$, $s_{j-2}\left(A_{n+2}\right)=A_{j}$ and $s_{j-2}\left(A_{a_{j}\left(r \cdot T^{\prime}\right)}\right)=A_{n+1+j-a_{j}\left(r \cdot T^{\prime}\right)}=A_{j-a_{j}\left(r \cdot T^{\prime}\right)-1}$ since $a_{j}\left(r \cdot T^{\prime}\right)<j$. Therefore $s_{j-2}\left(\Delta_{j}\left(r \cdot T^{\prime}\right)\right)=A_{j-a_{j}\left(r \cdot T^{\prime}\right)-1} A_{j} A_{n+1}=\Delta_{j}\left(s_{j-2} r \cdot T^{\prime}\right)$ and $\delta_{j}\left(s_{j-2} r \cdot T^{\prime}\right)=$ $\left(a_{j}\left(r \cdot T^{\prime}\right)+1\right)(n+1-j)$. Finally we obtain that

$$
\begin{aligned}
\delta_{j}\left(r \cdot T^{\prime}\right)+\delta_{j}\left(s_{j-2} r \cdot T^{\prime}\right) & =\left(j-a_{j}\left(r \cdot T^{\prime}\right)\right)(n+1-j)+\left(a_{j}\left(r \cdot T^{\prime}\right)+1\right)(n+1-j) \\
& =(j+1)(n+1-j) .
\end{aligned}
$$


Since $\left\{H, s_{k} H\right\}$ forms a partition of $\mathcal{D}_{n+2}$ for any $k$, we have

$$
\begin{aligned}
\sum_{\substack{f \in \mathcal{D}_{n+2}, a_{j+1}(f \cdot T)=0}} \delta_{j+1}(f \cdot T) & =\sum_{\substack{f \in H, a_{j+1}(f \cdot T)=0}}\left(\delta_{j+1}(f \cdot T)+\delta_{j+1}\left(s_{j} f \cdot T\right)\right) \\
& =\sum_{\substack{f \in H, a_{j+1}(f \cdot T)=0}}(j+1)(n+1-j) \\
& =\sum_{\substack{r f \in H, b_{j}(r f \cdot T)=n+1}}\left(\delta_{j}(r f \cdot T)+\delta_{j}\left(s_{j-2} r f \cdot T\right)\right), \text { since } r \in H \\
& =\sum_{\substack{g \in H, b_{j}(g \cdot T)=n+1}} \delta_{j}(g \cdot T) .
\end{aligned}
$$

We conclude the induction by adding Equations (1) and (2).

Proof of Theorem 2.2. We have to prove that

$$
\vec{u}=\sum_{T^{\prime} \in \mathcal{O}(T)} \overrightarrow{G M\left(T^{\prime}\right)}=\overrightarrow{0}
$$

Denote by $\operatorname{Stab}\left(T^{\prime}\right)=\left\{f \in \mathcal{D}_{n+2} \mid f \cdot T^{\prime}=T^{\prime}\right\}$ the stabilizer of $T^{\prime}$, then

$$
\sum_{f \in \mathcal{D}_{n+2}} M(f \cdot T)=\sum_{T^{\prime} \in \mathcal{O}(T)}\left|\operatorname{Stab}\left(T^{\prime}\right)\right| M\left(T^{\prime}\right) .
$$

Since $T^{\prime} \in \mathcal{O}(T),\left|\operatorname{Stab}\left(T^{\prime}\right)\right|=|\operatorname{Stab}(T)|=\frac{2(n+2)}{|\mathcal{O}(T)|}$, we have

$$
\sum_{f \in \mathcal{D}_{n+2}} M(f \cdot T)=\frac{2(n+2)}{|\mathcal{O}(T)|} \sum_{T^{\prime} \in \mathcal{O}(T)} M\left(T^{\prime}\right) .
$$

Therefore by Proposition 2.3 we have for any $i \in[n]$

$$
\sum_{T^{\prime} \in \mathcal{O}(T)} \delta_{i}\left(T^{\prime}\right)=\frac{|\mathcal{O}(T)|}{2(n+2)}(n+1)(n+2)=\frac{|\mathcal{O}(T)|(n+1)}{2} .
$$

Denote by $O$ the point of origin of $\mathbb{R}^{n}$. Then $\overrightarrow{O M}=M$ for any point $M$ of $\mathbb{R}^{n}$. By Chasles' relation we have finally

$$
\vec{u}=\sum_{T^{\prime} \in \mathcal{O}(T)} \overrightarrow{G M\left(T^{\prime}\right)}=\sum_{T^{\prime} \in \mathcal{O}(T)}\left(M\left(T^{\prime}\right)-G\right)=\sum_{T^{\prime} \in \mathcal{O}(T)} M\left(T^{\prime}\right)-|\mathcal{O}(T)| G .
$$

So the $i^{\text {th }}$ coordinate of $\vec{u}$ is $\sum_{T^{\prime} \in \mathcal{O}(T)} \delta_{i}\left(T^{\prime}\right)-\frac{|\mathcal{O}(T)|(n+1)}{2}=0$, hence $\vec{u}=\overrightarrow{0}$ by (3). 


\section{Center of gravity of generalized associahedra of type $A$ and $B$}

\subsection{Realizations of associahedra}

As a Coxeter group (of type $A$ ), $S_{n}$ is generated by the simple transpositions $\tau_{i}=(i, i+1$ ), $i \in[n-1]$. The Coxeter graph $\Gamma_{n-1}$ is then

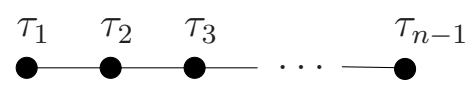

Let $\mathscr{A}$ be an orientation of $\Gamma_{n-1}$. We distinguish between up and down elements of $[n]$ : an element $i \in[n]$ is up if the edge $\left\{\tau_{i-1}, \tau_{i}\right\}$ is directed from $\tau_{i}$ to $\tau_{i-1}$ and down otherwise (we set 1 and $n$ to be down). Let $\mathrm{D}_{\mathscr{A}}$ be the set of down elements and let $\mathrm{U}_{\mathscr{A}}$ be the set of up elements (possibly empty).

The notion of up and down induces a labeling of the $(n+2)$-gon $P$ as follows. Label $A_{0}$ by 0 . Then the vertices of $P$ are, in counterclockwise direction, labeled by the down elements in increasing order, then by $n+1$, and finally by the up elements in decreasing order. An example is given in Figure 2.

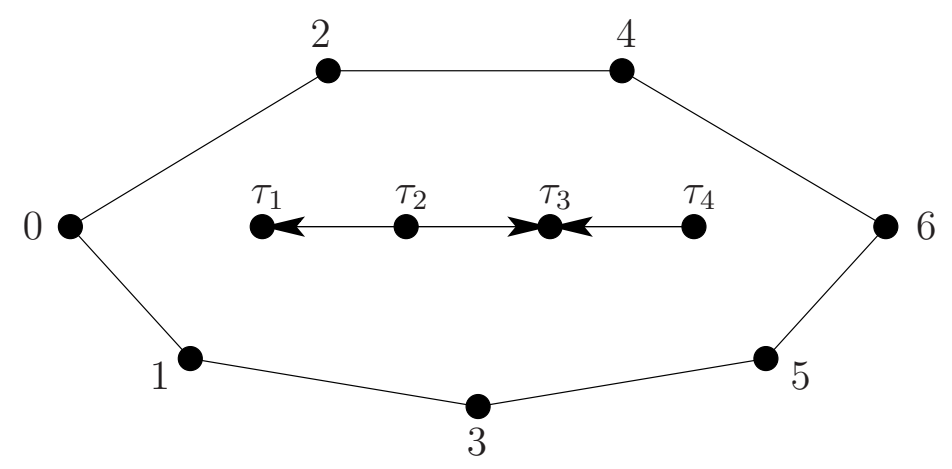

Figure 2: A labeling of a heptagon that corresponds to the orientation $\mathscr{A}$ of $\Gamma_{4}$ shown inside the heptagon. We have $\mathrm{D}_{\mathscr{A}}=\{1,3,5\}$ and $\mathrm{U}_{\mathscr{A}}=\{2,4\}$.

We recall here a construction due to Hohlweg and Lange [7]. Consider $P$ labeled according to a fixed orientation $\mathscr{A}$ of $\Gamma_{n-1}$. For each $l \in[n]$ and any triangulation $T$ of $P$, there is a unique triangle $\Delta_{l}^{\mathscr{A}}(T)$ whose vertices are labeled by $k<l<m$. Now, count the number of edges of $P$ between $i$ and $k$, whose vertices are labeled by smaller numbers than $l$. Then multiply it by the number of edges of $P$ between $l$ and $m$, whose vertices 
are labeled by greater numbers than $l$. The result $\omega_{l}^{\mathscr{A}}(T)$ is called the weight of $\Delta_{l}^{\mathscr{A}}(T)$. The injective map

$$
\begin{aligned}
M_{\mathscr{A}}: \mathcal{T}_{n+2} & \longrightarrow \mathbb{R}^{n} \\
T & \longmapsto\left(x_{1}^{\mathscr{A}}(T), x_{2}^{\mathscr{A}}(T), \ldots, x_{n}^{\mathscr{A}}(T)\right)
\end{aligned}
$$

that assigns explicit coordinates to a triangulation is defined as follows:

$$
x_{j}^{\mathscr{A}}(T):= \begin{cases}\omega_{j}^{\mathscr{A}}(T) & \text { if } j \in \mathrm{D}_{\mathscr{A}} \\ n+1-\omega_{j}^{\mathscr{A}}(T) & \text { if } j \in \mathrm{U}_{\mathscr{A}} .\end{cases}
$$

Hohlweg and Lange showed that the convex hull Asso $\mathscr{A}_{(}\left(S_{n}\right)$ of $\left\{M_{\mathscr{A}}(T) \mid T \in \mathcal{T}_{n+2}\right\}$ is a realization of the associahedron with integer coordinates [7, Theorem 1.1]. Observe that if the orientation $\mathscr{A}$ is canonic, that is, if $\mathrm{U}_{\mathscr{A}}=\emptyset$, then $\operatorname{Asso}_{\mathscr{A}}\left(S_{n}\right)=\operatorname{Asso}\left(S_{n}\right)$.

The key is now to observe that the weight of $\Delta_{j}^{\mathscr{A}}(T)$ in $T$ is precisely the weight of $\Delta_{j}\left(T^{\prime}\right)$ where $T^{\prime}$ is a triangulation in the orbit of $T$ under the action of $\mathcal{D}_{n+2}$, as stated in the next proposition.

Proposition 3.1. Let $\mathscr{A}$ be an orientation of $\Gamma_{n-1}$. Let $j \in[n]$ and let $A_{l}$ be the vertex of $P$ labeled by $j$. There is an isometry $r_{j}^{\mathscr{A}} \in \mathcal{D}_{n+2}$ such that:

(i) $r_{j}^{\mathscr{A}}\left(A_{l}\right)=A_{j}$;

(ii) the label of the vertex $A_{k}$ is smaller than $j$ if and only if the index $i$ of the vertex $A_{i}=r_{j}^{\mathscr{A}}\left(A_{k}\right)$ is smaller than $j$.

Moreover, for any triangulation $T$ of $P$ we have $\omega_{j}^{\mathscr{A}}(T)=\delta_{j}\left(r_{j}^{\mathscr{A}} \cdot T\right)$.

Proof. If $\mathscr{A}$ is the canonical orientation, then $r_{j}^{\mathscr{A}}$ is the identity, and the proposition is straightforward. In the following proof, we suppose therefore that $U_{\mathscr{A}} \neq \emptyset$.

Case 1: Assume that $j \in \mathrm{D}_{\mathscr{A}}$. Let $\alpha$ be the greatest up element smaller than $j$ and let $A_{\alpha+1}$ be the vertex of $P$ labeled by $\alpha$. Then by construction of the labeling, $A_{\alpha}$ is labeled by a larger number than $j$, and $\left[A_{\alpha}, A_{\alpha+1}\right]$ is the unique edge of $P$ such that $A_{\alpha+1}$ is labeled by a smaller number than $j$. Denote by $\Lambda_{\mathscr{A}}$ the path from $A_{l}$ to $A_{\alpha+1}$ passing through vertices of $P$ labeled by smaller numbers than $j$. This is the path going from $A_{l}$ to $A_{\alpha+1}$ in clockwise direction on the boundary of $P$.

By construction, $A_{k} \in \Lambda_{\mathscr{A}}$ if and only if the label of $A_{k}$ is smaller than $j$. In other words, the path $\Lambda_{\mathscr{A}}$ consists of all vertices of $P$ labeled by smaller numbers than $j$. Therefore the cardinality of $\Lambda_{\mathscr{A}}$ is $j+1$.

Consider $r_{j}^{\mathscr{A}}$ to be the rotation mapping $A_{l}$ to $A_{j}$. Recall that a rotation is an isometry preserving the orientation of the plane. Then the path $\Lambda_{\mathscr{A}}$, which is obtained by walking on the boundary of $P$ from $A_{l}$ to $A_{\alpha+1}$ in clockwise direction, is sent to the path $\Lambda$ obtained by walking on the boundary of $P$ in clockwise direction from $A_{j}$ and going through $j+1=\left|\Lambda_{\mathscr{A}}\right|$ vertices of $P$. Therefore $\Lambda=\left\{A_{0}, A_{1}, \ldots, A_{j}\right\}$, thus proving the first claim of our proposition in this case. 
Case 2: assume that $j \in \mathrm{U}_{\mathscr{A}}$. The proof is almost the same as in the case of a down element. Let $\alpha$ be the greatest down element smaller than $j$ and let $A_{\alpha}$ be the vertex of $P$ labeled by $\alpha$. Then by construction of the labeling, $A_{\alpha+1}$ is labeled by a larger number than $j$, and $\left[A_{\alpha}, A_{\alpha+1}\right]$ is the unique edge of $P$ such that $A_{\alpha}$ is labeled by a smaller number than $j$. Denote by $\Lambda_{\mathscr{A}}$ the path from $A_{l}$ to $A_{\alpha}$ passing through vertices of $P$ labeled by smaller numbers than $j$. This is the path going from $A_{\alpha}$ to $A_{l}$ in clockwise direction on the boundary of $P$.

As above, $A_{k} \in \Lambda_{\mathscr{A}}$ if and only if the label of $A_{k}$ is smaller than $j$. In other words, the path $\Lambda_{\mathscr{A}}$ consists of all the vertices of $P$ labeled by smaller numbers than $j$. Therefore, again, the cardinality of $\Lambda_{\mathscr{A}}$ is $j+1$.

Let $r_{j}^{\mathscr{A}}$ be the reflection mapping $A_{\alpha}$ to $A_{0}$ and $A_{\alpha+1}$ to $A_{n+1}$. Recall that a reflection is an isometry reversing the orientation of the plane. Then the path $\Lambda_{\mathscr{A}}$, which is obtained by walking on the boundary of $P$ from $A_{\alpha}$ to $A_{l}$ in clockwise direction, is sent to the path $\Lambda$ obtained by walking on the boundary of $P$ in clockwise direction from $A_{\alpha}$ and going through $j+1=\left|\Lambda_{\mathscr{A}}\right|$ vertices of $P$. Therefore $\Lambda=\left\{A_{0}, A_{1}, \ldots, A_{j}\right\}$. Hence $r_{j}^{\mathscr{A}}\left(A_{l}\right)$ is sent on the final vertex of the path $\Lambda$ which is $A_{j}$, proving the first claim of our proposition.

Thus it remains to show that for a triangulation $T$ of $P$ we have $\omega_{j}^{\mathscr{A}}(T)=\delta_{j}\left(r_{j}^{\mathscr{A}} \cdot T\right)$. We know that $\Delta_{j}^{\mathscr{A}}(T)=A_{k} A_{l} A_{m}$ such that the label of $A_{k}$ is smaller than $j$, which is smaller than the label of $A_{m}$. Write $A_{a}=r_{j}^{\mathscr{A}}\left(A_{k}\right)$ and $A_{b}=r_{j}^{\mathscr{A}}\left(A_{m}\right)$. Because of Proposition 3.1, $a<j<b$ and therefore

$$
r_{j}^{\mathscr{A}}\left(\Delta_{j}^{\mathscr{A}}(T)\right)=A_{a} A_{j} A_{b}=\Delta_{j}\left(r_{j}^{\mathscr{A}} \cdot T\right) .
$$

So $(j-a)$ is the number of edges of $P$ between $A_{l}$ and $A_{k}$, whose vertices are labeled by smaller numbers than $j$. Similarly, $(b-j)$ is the number of edges between $A_{l}$ and $A_{m}$, whose vertices are labeled by smaller numbers than $j$, and $(b-j)$ is the number of edges of $P$ between $A_{l}$ and $A_{m}$ and whose vertices are labeled by larger numbers than $j$. So $\omega_{l}^{\mathscr{A}}(T)=(j-a)(b-j)=\delta_{j}\left(r_{j}^{\mathscr{A}} \cdot T\right)$.

Corollary 3.2. For any orientation $\mathscr{A}$ of the Coxeter graph of $S_{n}$ and for any $j \in[n]$, we have

$$
\sum_{f \in \mathcal{D}_{n+2}} x_{j}^{\mathscr{A}}(f \cdot T)=(n+1)(n+2) .
$$

Proof. Let $r_{j}^{\mathscr{A}} \in \mathcal{D}_{n+2}$ be as in Proposition 3.1.

Suppose first that $j \in \mathrm{U}_{\mathscr{A}}$, then

$$
\begin{aligned}
\sum_{f \in \mathcal{D}_{n+2}} x_{i}^{\mathscr{A}}(f \cdot T) & =2(n+2)(n+1)-\sum_{f \in \mathcal{D}_{n+2}} \omega_{i}^{\mathscr{A}}(f \cdot T) \\
& =2(n+2)(n+1)-\sum_{f \in \mathcal{D}_{n+2}} \delta_{j}\left(f r_{j}^{\mathscr{A}} \cdot T\right), \text { by Proposition } 3.1 \\
& =2(n+2)(n+1)-\sum_{g \in \mathcal{D}_{n+2}} \delta_{j}\left(g^{\mathscr{A}} \cdot T\right), \text { since } r_{j}^{\mathscr{A}} \in \mathcal{D}_{n+2} \\
& =(n+1)(n+2), \text { by Proposition } 2.3
\end{aligned}
$$

If $i \in \mathrm{D}_{\mathscr{A}}$, the result follows from a similar calculation. 


\subsection{Center of gravity of associahedra}

Theorem 3.3. The center of gravity of $\operatorname{Asso}_{\mathscr{A}}\left(S_{n}\right)$ is $G=\left(\frac{n+1}{2}, \frac{n+1}{2}, \ldots, \frac{n+1}{2}\right)$ for any orientation $\mathscr{A}$.

By following precisely the same arguments as in $\S 2.3$, we just have to show the following generalization of Theorem 2.2.

Theorem 3.4. Let $\mathcal{O}$ be an orbit of the action of $\mathcal{D}_{n+2}$ on $\mathcal{T}_{n+2}$, then $G$ is the center of gravity of $\left\{M_{\mathscr{A}}(T) \mid T \in \mathcal{O}\right\}$. In particular, $\sum_{T \in \mathcal{O}} \overrightarrow{G M_{\mathscr{A}}(T)}=\overrightarrow{0}$.

Proof. The proof is entirely similar to the proof of Theorem 2.2, using Corollary 3.2 instead of Proposition 2.3.

\section{Center of gravity of the cyclohedron}

In 1994, R. Bott and C. Taubes discovered the cyclohedron [3] in connection with knot theory. It was rediscovered independently by R. Simion [20]. In [7], the first author and C. Lange also gave a family of realizations for the cyclohedron, starting with the permutahedron of type $B$. We show in this section that the centers of gravity of the cyclohedron and of the permutahedron of type $B$ are the same.

\subsection{The type $B$-permutahedron}

The hyperoctahedral group $W_{n}$ is defined by $W_{n}=\left\{\sigma \in S_{2 n} \mid \sigma(i)+\sigma(2 n+1-i)=\right.$ $2 n+1, \forall i \in[n]\}$. The type B-permutahedron Perm $\left(W_{n}\right)$ is the simple $n$-dimensional convex polytope defined as the convex hull of the points

$$
M(\sigma)=(\sigma(1), \sigma(2), \ldots, \sigma(n)) \in \mathbb{R}^{2 n}, \quad \forall \sigma \in W_{n} .
$$

As $w_{0}=(2 n, 2 n-1, \ldots, 3,2,1) \in W_{n}$, we deduce from the same argument as in the case of $\operatorname{Perm}\left(S_{n}\right)$ that the center of gravity of $\operatorname{Perm}\left(W_{n}\right)$ is

$$
G=\left(\frac{2 n+1}{2}, \frac{2 n+1}{2}, \ldots, \frac{2 n+1}{2}\right) .
$$

\subsection{Realizations of the associahedron}

An orientation $\mathscr{A}$ of $\Gamma_{2 n-1}$ is symmetric if the edges $\left\{\tau_{i}, \tau_{i+1}\right\}$ and $\left\{\tau_{2 n-i-1}, \tau_{2 n-i}\right\}$ are oriented in opposite directions for all $i \in[2 n-2]$. There is a bijection between symmetric orientations of $\Gamma_{2 n-1}$ and orientations of the Coxeter graph of $W_{n}$ (see $[7, \S 1.2]$ ). A triangulation $T \in \mathcal{T}_{2 n+2}$ is centrally symmetric if $T$, viewed as a triangulation of $P$, is centrally symmetric. Let $\mathcal{T}_{2 n+2}^{B}$ be the set of the centrally symmetric triangulations of $P$. In [7, Theorem 1.5] the authors show that for any symmetric orientation $\mathscr{A}$ of $\Gamma_{2 n-1}$. The convex hull Asso $\mathscr{A}_{(}\left(W_{n}\right)$ of $\left\{M_{\mathscr{A}}(T) \mid T \in \mathcal{T}_{2 n+2}^{B}\right\}$ is a realization of the cyclohedron with integer coordinates. 
Since the full orbit of symmetric triangulations under the action of $\mathcal{D}_{2 n+2}$ on triangulations provides vertices of Asso $\mathscr{A}\left(W_{n}\right)$, and vice-versa, Theorem 3.4 implies the following corollary.

Corollary 4.1. Let $\mathscr{A}$ be a symmetric orientation of $\Gamma_{2 n-1}$, then the center of gravity of Asso $_{\mathscr{A}}\left(W_{n}\right)$ is $G=\left(\frac{2 n+1}{2}, \frac{2 n+1}{2}, \ldots, \frac{2 n+1}{2}\right)$.

\section{References}

[1] N. Bergeron, C. Hohlweg, C. Lange and H. Thomas, Isometry classes of generalized associahedra Séminaire Lotharingien de Combinatoire, B61Aa (2009), 13 pp.

[2] J. M. BLOom, A link surgery spectral sequence in monopole Floer homology, arXiv: math.CO/0909.0816.

[3] R. Bott and C. Taubes, On the self-linking of knots, J. Math. Phys. 35 (1994), $5247-5287$.

[4] F. Chapoton, S. Fomin And A. Zelevinsky, Polytopal realizations of generalized associahedra, Canad. Math. Bull. 45 (2003), 537-566.

[5] S. Fomin And A. Zelevinsky, Y-systems and generalized associahedra, Annals of Mathematics 158 (2003), 977-1018.

[6] S. Forcey, A. Lauve, F. Sottile, New Hopf structures on binary trees, arXiv: math.CO/0708.3709.

[7] C. Hohlweg and C. Lange, Realizations of the Associahedron and Cyclohedron, Discrete Comput Geom 37 (2007), 517-543.

[8] C. Hohlweg, C. Lange, H. Thomas, Permutahedra and Generalized Associahedra, arXiv:math.CD/0709.4241.

[9] J.-L. Loday, Realization of the Stasheff polytope, Arch. Math. 83 (2004), 267-278.

[10] J.-L. LodAy, The diagonal of the Stasheff polytope, arXiv:math.C0/0710.0572.

[11] B. Mirtich, Fast and Accurate Computation of Polyhedral Mass Properties, Journal of Graphics Tools v.1 n.2 (1996).

[12] R. Marsh, M. Reineke and A. Zelevinsky, Generalized associahedra via quiver representations. Trans. Amer. Math. Soc. 355 (2003) no. 10, 4171-4186.

[13] A. Postnikov, Permutohedra, associahedra, and beyond (2004), 59 pages, arXiv: math. CO/0507163.

[14] N. Reading, Cambrian lattices, Adv. in Mathematics 205(2) (2006), 313-353.

[15] N. Reading, Clusters, Coxeter-sortable elements and noncrossing partitions, Trans. Am. Math. Soc. 359(12) (2007), 5931-5958.

[16] N. Reading, Sortable elements and Cambrian lattices, Algebra Universalis 56(3-4) (2007), 411-437. 
[17] N. Reading And D. Speyer, Cambrian fans (2006), to appear in J. Eur. Math. Soc., 40 pages, arXiv:math.C0/0606201.

[18] M. I. Shamos And F. P. Preparata, Computational Geometry, Springer-Verlag (1985).

[19] S. Shnider And S. Sternberg, Quantum groups: From coalgebas to Drinfeld algebras, Graduate texts in mathematical physics, International Press, 1994.

[20] R. Simion, A type-B associahedron, Adv. Appl. Math. 30 (2003), 2-25.

[21] J. Stasheff, Homotopy associativity of H-spaces I, II, Trans. Amer. Math. Soc. 108 (1963), 275-312.

[22] J. StashefF, From operads to "physically" inspired theories, Operads: Proceedings of Renaissance Conferences (Hartford, CT/Luminy, 1995), 53-81, Contemp. Math., 202, Americ. Math. Soc. (1997).

[23] G. ZiegleR, Lectures on polytopes, Springer-Verlag, New York, 1995. 